\title{
The Human Mycobiome
}

\author{
Patrick C. Seed \\ Departments of Pediatrics, Molecular Genetics and Microbiology, and Surgery, \\ Duke University School of Medicine, Durham, North Carolina 27710 \\ Correspondence: patrick.seed@duke.edu
}

Fungi are fundamental to the human microbiome, the collection of microbes distributed across and within the body, and the microbiome has been shown, in total, to modify fundamental human physiology, including energy acquisition, vitamin-cofactor availability, xenobiotic metabolism, immune development and function, and even neurological development and behavior. Here, a comprehensive review of current knowledge about the mycobiome, the collective of fungi within the microbiome, highlights methods for its study, diversity between body sites, and dynamics during human development, health, and disease. Earlystage studies show interactions between the mycobiome and other microbes, with host physiology, and in pathogenic and mutualistic phenotypes. Current research portends a vital role for the mycobiome in human health and disease.

$\mathrm{T}_{\mathrm{i}}^{\mathrm{h}}$ he collection of microbes across and within the human body modifies inflammation and immunity while providing a constellation of physiological and metabolic functions not otherwise innate in human physiology. As such, the microbes in aggregate, the so-called human microbiome, act as modifiers of fundamental human physiology, including energy acquisition, vitamin-cofactor availability, xenobiotic metabolism, immune development and function, and even neurological development and behavior (Pflughoeft and Versalovic 2012).

Although the composition and dynamics of the bacterial constituents of the microbiome have been the focus of extensive studies in health and disease, other members of the microbiome are much less well characterized. These include fungi, viruses, and parasites/protozoa. In particular, the diversity and dynamics of the so-called mycobiome, the fungi distributed on and within the body, is poorly understood, particularly in light of the considerable association of fungi with infectious diseases and allergy (Walsh and Dixon 1996). Despite being as low as $\leq 0.1 \%$ of the total microbiota (Qin et al. 2010), the fungal constituents of the microbiome may have key roles in maintaining microbial community structure, metabolic function, and immune-priming frontiers, which remain relatively unexplored. Further questions exist as to how fungi interact cooperatively and noncooperatively with nonfungal constituents of the microbiome.

Here, multiple facets of the study of the mycobiome are reviewed, beginning with an overview of the methodologies used to describe the constitution of the mycobiome and to study its phenotype. Current knowledge of

Editors: Arturo Casadevall, Aaron P. Mitchell, Judith Berman, Kyung J. Kwon-Chung, John R. Perfect, and Joseph Heitman

Additional Perspectives on Human Fungal Pathogens available at www.perspectivesinmedicine.org

Copyright (C) 2015 Cold Spring Harbor Laboratory Press; all rights reserved; doi: 10.1101/cshperspect.a019810

Cite this article as Cold Spring Harb Perspect Med 2015;5:a019810 
P.C. Seed

the mycobiome in health and during specific states of disease is appraised, and critical questions for future studies of the mycobiome are proffered.

\section{MAPPING THE MYCOBIOME}

The characterization of the microbiome and the mycobiome within it begins with sample collection. In the case of mycobiome analysis, sample collection has several challenges. First, fungi may be present in human-associated niches at much lower abundance than bacteria, which are plentiful throughout the enteric tract, on the skin, and in the vagina. Second, human cells (or model animal cells) may contaminate samples and interfere with specific amplification and sequencing of fungal sequences, such as ribosomal RNA (rRNA). Third, isolation of quality genetic material from fungi may be more challenging than from many bacteria or animal cells. These issues must all be considered when planning protocols for sample collection.

For the skin mycobiome, scrapings and swabs have been used, focusing on the wide variety of dry, moist, and oily sites distributed across the body (Park et al. 2012; Findley et al. 2013). For the oral mycobiome, sample collection may include oral washes from which cells may be collected by centrifugation (Ghannoum et al. 2010; Mukherjee et al. 2014). Gingival and buccal (cheek) scrapings are also complementary approaches for sample collection (Wade 2013). For the human intestinal mycobiome, feces are most frequently used as a convenient and noninvasive sample type from which to obtain an overview of the types of organisms present in the intestinal tract (LaTuga et al. 2011; Hoffmann et al. 2013). In some circumstances, bowel biopsies or surgical samples (oral cavity through to rectum) may be available and processed to identify mucosalassociated organisms, which may be disproportionately represented in the feces (Araújo-Pérez et al. 2012; Huse et al. 2014). The lung may be sampled through induced deep sputum samples or endoscopy and bronchoalveolar lavage (BAL), in which the airways are directly visualized, washed with saline, and sampled by vacu- um suctioning of an applied wash (CabreraRubio et al. 2012; Lynch and Bruce 2013). Paired oral washes and BAL samples are advisable to distinguish oral contamination of deep lung collections from the lung-associated organisms. Extraction of fungal nucleic acids from biological samples may entail rigorous physical methods to lyse the organisms. Bead beating and enzymatic combinations have been used for this purpose. In general, protocols and commercial reagent kits for soil microbial nucleic acid extractions have been sufficient, although a head-to-head comparison of different extraction methods to optimize the study of the mycobiome has not been published to date.

Molecular identification of fungal species present within biological samples has primarily relied on high-throughput, "next-generation" sequencing of rRNA regions. Two regions have been most frequently used for these studies. First, the 18S rRNA has been used in sequencing (Dollive et al. 2012). Second, regions internal to the $18 \mathrm{~S}, 5.8 \mathrm{~S}$, and $28 \mathrm{~S}$ rRNA sequences termed the internal transcribed spacer regions (ITS) have been sequenced (White et al. 2013). Reference databases have been established for each sequence type (Kõljalg et al. 2005; Pruesse et al. 2007; Mahé et al. 2012; Santamaria et al. 2012; Hoffmann et al. 2013; White et al. 2013). Each region targeted for sequencing may be amplified using primers designed to hybridize to conserved sequences among most fungi with variable regions between the primer sites that have sequence variations specific to different groups of taxa. The polymerase chain reaction (PCR) may be used to amplify the variable region using primers directed against the conserved flanking regions. In most circumstances, the template is total genomic DNA, which has the advantage of greater stability compared with rRNA and less bias because of rRNA expression levels. Multiple sequencing technologies have been used for sequencing the mixtures of amplicons obtained from PCR of different samples. These include Roche 454, Illumina HiSeqMiSeq, and Ion Torrent (Di Bella et al. 2013). The sequencing information obtained from these platforms produces millions to trillions of sequences, which the investigative group 
must be prepared to store and process in an informatics analysis. Although it is beyond the scope of this review, there are a growing number of reference databases and analysis tools to use for assignment of taxa to the sequences and compare sample composition between samples and study groups (Cole et al. 2003; Kõljalg et al. 2005; Pruesse et al. 2007; Schloss et al. 2009; Caporaso et al. 2010; Mitra et al. 2011; White et al. 2013).

The constitution of the microbiome may also be determined through sequencing and analysis of total genomic DNA, or transcripts in processes called shotgun metagenomics and metatranscriptomics (Qin et al. 2010; Gilbert and Hughes 2011). For bacteria, phage, and viruses, whole genomes have been reassembled from sequences obtained by shotgun metagenomics. In so doing, function may be inferred for different organisms; however, as a result of the low abundance of fungi in most biological specimens, these approaches have proven challenging and have yet to produce data sets with sufficient sequence data to assemble the genomes of the fungi from complex samples like human feces (Qin et al. 2010).

Despite the power of next-generation sequencing to detail the membership of fungi within the microbiome as a whole, culturebased methods remain important. Because of the low abundance of many fungi within complex microbial communities, selective culture methods may be required to isolate fungi of interest, sequence their genomes, and perform functional analyses, including studies of metabolism, host interactions, and antibiotic susceptibility.

\section{DEVELOPMENT OF THE MYCOBIOME}

Over the past decade, the microbiome has been shown to broadly alter the physiology of its host, including metabolism, immunity, and even neurological development. Thus, there has been strong interest in understanding the assembly of the microbiome in the developing animal, as early life configurations appear to herald later stages of health and disease. To date, the study of the naturally assembling microbiome, particu- larly in humans, remains early stage and has been largely focused on bacterial constituents within the larger microbiome, with much less effort in understanding the diversity and timing of assembling fungi into the microbiome and their contribution to the microbiome phenotype on the whole.

The prevailing view is that the healthy full-term infant is born sterile, although recent evidence is challenging this notion (EstradaGutierrez et al. 2010; Aagaard et al. 2014; Cao and Mysorekar 2014). Although it is outside the scope of this paper to debate this theory, the passage of the full-term infant through the vaginal canal is likely the first major exposure to the microbial world from which the earliest colonizers of the infant are derived. Interestingly, although the bacterial components of the vaginal microbiome have been the subject of intensive study (Ravel et al. 2011; Ma et al. 2012), the fungal components of the vaginal microbiome are primarily understood through culture-based methods and only a single large culture-independent study of nonpregnant Estonian women (discussed later) (Drell et al. 2013). Because of this limitation, a discussion of the degree to which the vaginal mycobiome alters infant colonization is not yet feasible.

Fungal colonization of the term infant remains poorly characterized. Although it is known that fungi, such as Candida, are prevalent constituents of the vagina through which most infants are delivered, transmission to the newborn is not well documented, and assembly of additional environmental fungi into the microbiome has not been monitored in the otherwise healthy infant. Previously, in a microarray-based, culture-independent study of 10 infants' microbiomes during the first $18 \mathrm{mo}$ of life, investigators performed general PCR-based quantification of fungi from infant stool samples using PCR primers to the small subunit rRNA (Palmer et al. 2007). Here, variable levels of fungi were identified in samples throughout the study. Two circumstances of interest were noted; fungal sequences were identified in the vaginal sample of a mother who had a vaginal birth of a healthy infant who did not have fun- 
P.C. Seed

gal sequences in the initial stool samples. In this case, the presence of fungi in the vaginal microbiota did not necessarily result in significant colonization of the infant. Of course, these data are limited because of the single case and lack of specific taxonomic information about the fungi in the vaginal microbiota. Of the infants with the most abundant and consistent fungal sequences amplified from stool, one developed a Candida-type diaper rash consistent with fungal overgrowth in the microbiota.

Although the microbiome of the healthy term infant remains poorly understood, more effort has been placed on understanding fungal colonization of preterm infants. Infants born 8 or more weeks before term and weighing $\leq 1500 \mathrm{~g}$ at birth are at significantly increased risk for invasive fungal disease, primarily with Candida species (spp.). In addition to prematurity of intestinal and systemic immunity, multiple factors put these infants at risk of Candida colonization and infection. First, these infants are likely to have broad antibiotic exposure before and following birth. Second, they are more likely to be delivered by caesarian section and have delayed feeding. Finally, culture-based surveillance data suggest $>50 \%$ of these very preterm infants have intestinal colonization with Candida spp. within the first 6 wk of life (Kaufman et al. 2006).

In a recent study, LaTuga et al. (2011) investigated the broader microbiome of the preterm infant, including bacteria and fungi. Among 11 infants born weighing $<1500 \mathrm{~g}$, seven had stool samples from which fungal ITS amplification was successful. The infant stool had a diverse fungal constituency even by 2 wk of age. The most abundant order was Saccharomycetales with at least six different predicted species of Candida, four different predicted species of Cladosporium, three different predicted species of Cryptococcus, and Saccharomyces cerevisiae. Also present in most samples were Malasseziales, Eurotiales, Botryosphaeriales, and Filobasidiales. Although this study highlights remarkable fungal diversity within the intestinal microbiome at an early age among preterm infants, it was limited by the small number of samples and lacked serial sampling from which the assembling mycobiome and its dynamic nature would be observed.

\section{THE MYCOBIOME IN HEALTH AND DISEASE}

\section{The Oral Mycobiome and HIV}

The oral cavity is the major portal of entry into the enteric and respiratory tracts. Its intersection with the nasal passageway and diverse microniches in the postdentition human leads to a complex environment for microbial community formation, geodiversity, mixing effects, and dynamic changes in response to environmental perturbations. The oral environment may filter incoming microbes, and its microbes may alter local and systemic innate and adaptive immunity.

Based on culture-dependent or genus/species-focused culture-independent methods of identification, the fungi of the oral cavity were previously believed to be few and relatively nondiverse. The genera Candida, Saccharomyces, Penicillium, Aspergillus, Scopulariopsis, and Genotrichum were among those previously reported (Schuster 1999; Salonen et al. 2000; Williams and Lewis 2000; Jabra-Rizk et al. 2001). Additional studies of oral mycoses indicate the presence of additional pathogenic genera including Cryptococcus, Histoplasma, and Mucor, among others (Iatta et al. 2009). In most circumstances, however, these studies were limited not only by reliance on culture or narrow PCR methods of identification, but they were also performed in otherwise immunocompromised individuals, such as those with HIV-AIDS or oncological processes in which the oral microbiota would be expected to be altered. Thus, until relatively recently, the oral mycobiome in the state of health using culture-independent methods was not available.

Addressing the gap in knowledge about the extent of the oral mycobiome, Ghannoum et al. (2010) used the ITS sequencing with Roche 454 (Branford, CT) technology to elucidate the oral microbiome in healthy adult subjects. In the oral samples from 20 participants, most had $\sim 15$ fungal genera present. Only one individual had more than 20 different fungal genera. 
To put this level of diversity into context, prior studies have identified more than $50-100$ bacterial genera in the healthy oral microbiome (Zaura et al. 2009; Bik et al. 2010).

Of the oral fungal genera noted among each of the healthy subjects from the Ghannoum study (Ghannoum et al. 2010), Candida and Cladosporium were most common, present in $75 \%$ and $65 \%$ of participants, respectively. Fungal genera associated with local oral and invasive diseases, including Aspergillus, Cryptococcus, Fusarium, and Alternaria were also identified, indicating that these genera are present in the oral microbiome even during a state of health.

Although the number of participants was small, the Ghannoum study points toward potential racial, ethnic, and gender factors in variation of the oral mycobiome (Ghannoum et al. 2010). Caucasian and Asians were overrepresented among the study participants; however, principle component analysis (PCoA) suggested different communities among Caucasian and Asian males, whereas females, independent of race or ethnicity, tended to cocluster more closely together. Follow-up studies will be required to determine whether these differences observed by PCoA are fundamentally a result of the host or environmental differences, including nutrition or smoking, for instance.

In the Ghannoum study (Ghannoum et al. 2010), all of the subjects had oral fungal genera categorized as "noncultivatable" and not previously taxonomically classified. In $30 \%$ of the participants, the noncultivatable group constituted $50 \%$ or more of the fungi identified. As noncultivatable organisms without broader genomic data for the respective organisms, their metabolic and physiologic capacity and interactions with the microbial community cannot be predicted. The discovery of previously unidentified fungi is a reminder that the oral microbiome remains underexplored.

Dupuy et al. (2014) also recently performed a study of the oral mycobiome in healthy adults. Using the well-curated Fungal Metagenomics Project ITS sequence database, they confirmed most of the taxa found in the study by Ghannoum et al. (2010); however, there were nota- ble differences, particularly in the most abundant taxa. In contrast to the Ghannoum study (Ghannoum et al. 2010), Dupuy et al. (2014) identified the Malassezia and Epicoccum as the most abundant genera at $38 \%$ and $33 \%$ of the total fungi, respectively. Candida, Cladosporium, and Alternaria were among the next most abundant genera, constituting 9.5\%, 3\%, and $\sim 2 \%$, respectively. Candida, Cladosporium, Alternaria, Aspergillus, Fusarium, Cryptococcus, and Aureobasidium were common in both of the studies, likely representing members of the core oral fungi in the healthy mycobiome. Malassezia, Irpex, Cytospora, Lenziles, and Sporobolomyces were uniquely represented in Dupuy et al. (2014), whereas Dothioraceae, Teratosphaeria, and Glomus were among the core taxa in Ghannoum et al. (2010). Sample collection and processing methods were similar and do not explain the observed differences between the two studies. These differences likely represent variation in the curation of the respective databases used for each study and, to some extent, may represent differences in the subjects involved in each study.

The association of the oral mycobiome and disease are currently under investigation. In a recent study by Mukherjee et al. (2014), the oral microbiome was compared with HIVinfected and uninfected individuals. Oral infections with Candida spp. are a well-known complication of HIV infection and may precede invasive infections of the esophagus, or even the bloodstream. Remarkably, the core bacterial taxa were similar between the two subject groups, whereas the fungal taxa differed significantly, suggesting differences in innate immune and adaptive cellular immunity in the constitution of the oral mycobiome. In particular, Candida, Aspergillus, and Fusarium were more abundant among the HIV-infected subjects. $P i$ chia and Candida abundance had a reciprocal relationship. In functional studies, spent growth medium from Pichia cultures was inhibitory to Candida hyphae formation and invasive disease in a murine model of Candida infection. The presence of oral Candida had a positive association with oral Atopobium, Capnocytophagia, and Fusobacterium. Pichia did not cor- 
P.C. Seed

relate negatively with these bacteria, suggesting that changes in these bacteria are not likely intermediates to the reduction of Candida in the Pichia-abundant oral mycobiome.

\section{The Lung Mycobiome and Lung Transplantation}

Contrary to conventional thought, recent studies have shown that the respiratory tree is not sterile (Hilty et al. 2010). Even the lower respiratory tract has a complex dynamic microbiome that is altered during diseases, such as cystic fibrosis and chronic obstructive pulmonary disease (Pragman et al. 2012). Despite the extensive knowledge of the bacterial constituency in the lung during states of health and some pulmonary diseases, knowledge of the fungal constituency lags severely behind.

A recent study of BAL samples collected from healthy individuals and lung transplant patients provides some preliminary understanding of the lung mycobiome in healthy and immunocompromised individuals (Charlson et al.2012). Oral and BAL washes were compared with healthy controls and lung transplant patients. Overall, oral ITS sequences were of similar abundance in both groups; however, controls had much lower ITS sequence abundance in the BAL samples compared with the transplant subjects. Control subjects had Aspergillus, Penicillium, and Davidiellaceae shared between the oral and BAL samples. In contrast, the BAL samples from lung transplant recipients had abundant fungal sequences. Candida spp. were moderate to high in abundance in $62 \%$ and $42 \%$ of the oral wash and BAL samples, respectively. In each case that Candida was abundant in the BAL, it was also abundant in that individual's oral wash sample. Only several BAL samples had a moderate to high abundance of Aspergillus and Penicillium; the fungi were otherwise rare or undetectable in the BAL fluid. When Aspergillus was detected in the BAL fluid of lung transplant patients, it was rare or not detected in the oral wash from the same individual.

Principle coordinate analysis using phylogenetic and nonphylogenetic metrics showed that the BAL sample was most similar to the oral wash sample from the same individual, mostly dictated by the bacterial constituency in each sample but supporting the notion that most of the lung microbiome arises from the oral cavity. The lung transplant patients' oral wash and BAL sample communities were at greater distances from each other compared with intraindividual comparisons of control subject samples, further suggesting an outgrowth of oral microbiota in the transplanted lung environment within the immunocompromised host.

\section{The Intestinal Mycobiome, Inflammatory Bowel Disease, and Clostridium difficile Diarrhea}

The intestinal microbiome contains the greatest quantity and diversity of the human microbiome with an estimated one trillion organisms (Qin et al. 2010). The intestinal microbiome has been implicated in local and systemic metabolism, immune maturation and function, animal behavior, and diseases of these different systems. Although the bacterial constituents of the gut-associated microbiome have been intensely studied, the diversity and function of gut-associated fungi is understudied and lags far behind other aspects of microbiome studies.

Only recently have larger studies specifically focused on the gut mycobiome been performed. Hoffmann et al. (2013) recently performed deep sequencing of the ITS1 region for identification of fungi in fecal samples from 98 healthy individuals without known gastrointestinal disease. In total, the researchers identified 66 fungal genera with 13 additional taxa for which a genus-level classification was not possible. An estimated 184 species were present in total. Eighty-nine percent of the samples had Saccharomyces present. Candida and Cladosporium were the second and third most prevalent, present in $57 \%$ and $42 \%$ of samples, respectively. The research was not able to definitively determine whether certain taxa were resident fungal microbota or transient as part of dietary intake. For example, sequences for Agaricus, the white button mushroom, were identified in the feces 
and presumed to be derived from mushroom consumption, and Saccharomyces were abundant but may have come from dietary sources or may have been part of the resident microbiota.

Hoffmann et al. (2013) evaluated correlations between fungal and bacterial taxa in the gut microbiota. They showed that Candida and Saccharomyces were positively associated with the archea Methanobrevibacter and the bacteria Prevotella (Hoffmann et al. 2013). This group of organisms was most abundant among individuals with high carbohydrate diets. In contrast, those individuals with diets rich in amino acids and fatty acids had lower levels of Candida per stool. The investigators suggest that Candida, Prevotella, Ruminococcus, and Methanobrevibacter may form a syntrophic guild with Candida providing metabolisms of complex carbohydrates, whereas Prevotella and Ruminococcus ferment the more simple sugars liberated by Candida. In turn, Methanobrevibacter may consume the bacterial fermentation products to produce methane and carbon dioxide.

Mutualism between fungi and humans is generally not well understood and has not been well studied. However, several examples related to the gut microbiome provide evidence of a beneficial relationship. S. boulardii, closely related to Saccharomyces cerevisiae, has been studied in controlled trials for the prevention and mitigation of antibiotic-associated diarrhea, including diarrhea caused by Clostridium difficile. Trial data indicate that $S$. boulardii is able to decrease the likelihood of antibiotic-associated diarrhea and developing $C$. difficile diarrhea, and reduce the duration of diarrhea (Johnston et al. 2006; Tung et al. 2009). $S$. boulardii has been shown to stimulate antiC. difficile toxin IgA production and gut antiinflammatory pathways (Qamar et al. 2001; Pothoulakis 2009). These studies show the potency of fungi to compete with pathogenic organisms, modify intestinal function, and attenuate inflammation, presumably because of an interaction with the intestinal microbiota, although the consequences of $S$. boulardii consumption on the function and membership of the gut-associated microbiome has not been studied. A recent retrospective data review suggested an inverse relationship between Candida and $C$. difficile, pointing to some common impact of yeast on the gut microbiome and the exclusion of $C$. difficle outgrowth and/or toxin production (Manian and Bryant 2013).

Initiation and exacerbation of inflammatory bowel diseases, including Crohn's disease and ulcerative colitis, are believed to be incited and exacerbated by the gut microbiota (Pothoulakis 2009; Chow et al. 2011). A recent study suggested that a mutation in DECTIN-1, encoding for the widely expressed major fungal recognition C-type lectin receptor, is associated with human ulcerative colitis (Iliev et al. 2012). In wild-type mice, the investigators showed a progressively increasing abundance of fungal rDNA from the proximal to distal enteric tract. They showed that an adaptive antibody response against fungi developed following an inflammatory insult to the intestines. DECTIN-1 knockout mice $\left(\mathrm{Clec} a^{-/-}\right)$had enhanced tissue inflammation scores and heightened TNF- $\alpha$, IL-17, and IFN- $\gamma$ responses in the intestinal tract after an inflammatory stimulus. Antifungal therapy ameliorated inflammation in the DECTIN1 knockout animals, suggesting that the Dectin1 receptor is required to maintain inflammation homeostasis in the presence of a fungi-containing microbiota.

The role of Dectin-1 in controlling gut fungi and fungal-induced inflammation is complicated by additional studies. In contrast to Iliev et al. (2012), Tang et al. (2012) found that DECTIN-1 mice had attenuated inflammation following an inflammatory insult to the intestinal tract. Subsequently, studies of mice with macrophage-lineage-specific loss of Dectin-1 had lower inflammatory cytokine responses to fungi (Heinsbroek et al. 2012); however, they remained susceptible to inflammatory stress of the intestinal tract and were able to develop colitis, suggesting redundant pathways for the detection of fungi in the intestinal tract. Further study of Dectin-1 expression in inflamed bowel tissue from human subjects with ulcerative colitis did show increased Dectin-1 expression at the sites of inflammation, but did not corroborate DECTIN-1 c.714T > G allele frequencies 
P.C. Seed

previously proposed to be associated with inflammatory bowel disease (Iliev et al. 2012; Moyes and Naglik 2012). Together, these studies of Dectin in the control of fungal colonization and invasion show its importance; however, the physiological context and its role in mediating the host interaction with the mycobiome remains to be further refined and are exciting areas for future research.

\section{Vaginal Mycobiome and Candida Vaginosis}

The vaginal microbiome is a rich community and is well studied with regard to bacterial constituents (Ma et al. 2012). Although Candida is a common cause of vaginitis, the vaginal mycobiome is only recently being studied. In a recent study of the vaginal microbiome of 294 healthy Estonian women of reproductive age, ITS1-2 sequencing was performed on 251 samples to elucidate the fungal composition of the vaginal microbiome. Fifty-eight percent of the constituents were Ascomycota with $37 \%$ being Saccharomycetales, among which 16 different original taxonomic units of Candida spp. were most abundant (34\% of total). Additional Ascomycota Davidiellaceae, Cladosporium, Eurotium, Alternaria, and the basidomycota Rhodotorula, were present as only $1 \%$ of the total. A large percentage of the total sequences were unclassified within the fungal kingdom.

Regression analysis to associate vaginal fungal composition with medical history and lifestyle features was inconclusive. Although vaginal $\mathrm{pH}$ and discharge status correlated with the bacterial community composition, these and other features did not correlate with the fungal composition. In total, this study was not able to identify bacterial community or health features that may underlie the fungal composition in the vagina. Further studies will be needed to identify dysbiotic states that permit Candida overgrowth and alter mucosal inflammation.

\section{PROSPECTUS}

Humans have a lifelong interaction with complex microbial communities distributed across the body, which fundamentally contributes to the development and physiology of the mac- ro-organism. Only recently has the diversity of fungi within the human microbiome begun to be determined, with early studies showing that, although relatively nonabundant, fungi are diverse within the microbiome as a whole. Although still in the early stage, studies suggest complex interactions between fungal and bacterial constituents of the microbiome.

Numerous questions remain regarding the mycobiome. The early life development and maintenance of the mycobiome remains to be elucidated as does its susceptibility to perturbations, including nutritional stresses, metabolic alterations, such as diabetes and obesity, host inflammatory disorders, and xenobiotic exposure, including microbe-directed agents, such as anti-infectives, and host-directed agents, including agents chronically used for disease prevention, such as statins, aspirin, and others.

Albeit relatively rare within the microbiome, fungi have established roles in immune stimulation and dysregulation in conditions, such as in allergy and asthma. Much work remains to understand how fungi interact with the innate and adaptive immune system in the context of the microbiome.

Perhaps the most critical studies will be to understand the syntrophic guilds formed between fungi and bacteria within the microbiome. Through these studies, research will reveal the roles of fungi in community stability and resilience. These studies will also reveal how fungi work within complex microbial communities to contribute to energy harvest, metabolic homeostasis, and the balance of immunity, inflammation, and immunological tolerance.

\section{REFERENCES}

Aagaard K, Ma J, Antony KM, Ganu R, Petrosino J, Versalovic J. 2014. The placenta harbors a unique microbiome. Sci Transl Med 6: 237ra65.

Araújo-Pérez F, McCoy AN, Okechukwu C, Carroll IM, Smith KM, Jeremiah K, Sandler RS, Asher GN, Keku TO. 2012. Differences in microbial signatures between rectal mucosal biopsies and rectal swabs. Gut Microbes 3: 530-535.

Bik EM, Long CD, Armitage GC, Loomer P, Emerson J, Mongodin EF, Nelson KE, Gill SR, Fraser-Liggett CM, Relman DA. 2010. Bacterial diversity in the oral cavity of 10 healthy individuals. ISME J 4: 962-974. 
Cabrera-Rubio R, Garcia-Núñez M, Setó L, Antó JM, Moya A, Monsó E, Mira A. 2012. Microbiome diversity in the bronchial tracts of patients with chronic obstructive pulmonary disease. J Clin Microbiol 50: 3562-3568.

Cao B, Mysorekar IU. 2014. Intracellular bacteria in placental basal plate localize to extravillous trophoblasts. Placenta 35: 139-142.

Caporaso JG, Kuczynski J, Stombaugh J, Bittinger K, Bushman FD, Costello EK, Fierer N, Pena AG, Goodrich JK, Gordon JI, et al. 2010. QIIME allows analysis of highthroughput community sequencing data. Nat Methods 7: 335-336.

Charlson ES, Diamond JM, Bittinger K, Fitzgerald AS, Yadav A, Haas AR, Bushman FD, Collman RG. 2012. Lungenriched organisms and aberrant bacterial and fungal respiratory microbiota after lung transplant. Am J Respir Crit Care Med 186: 536-545.

Chow J, Tang H, Mazmanian SK. 2011. Pathobionts of the gastrointestinal microbiota and inflammatory disease. Curr Opin Immunol 23: 473-480.

Cole JR, Chai B, Marsh TL, Farris RJ, Wang Q, Kulam SA, Chandra S, McGarrell DM, Schmidt TM, Garrity GM, et al. 2003. The Ribosomal Database Project (RDP-II): Previewing a new autoaligner that allows regular updates and the new prokaryotic taxonomy. Nucleic Acids Res 31: 442-443.

Di Bella JM, Bao Y, Gloor GB, Burton JP, Reid G. 2013. High throughput sequencing methods and analysis for microbiome research. J Microbiol Methods 95: 401-414.

Dollive S, Peterfreund GL, Sherrill-Mix S, Bittinger K, Sinha R, Hoffmann C, Nabel CS, Hill DA, Artis D, Bachman MA, et al. 2012. A tool kit for quantifying eukaryotic rRNA gene sequences from human microbiome samples. Genome Biol 13: R60.

Drell T, Lillsaar T, Tummeleht L, Simm J, Aaspõllu A, Väin E, Saarma I, Salumets A, Donders GGG, Metsis M. 2013. Characterization of the vaginal micro- and mycobiome in asymptomatic reproductive-age Estonian women. PLoS ONE 8: e54379.

Dupuy AK, David MS, Li L, Heider TN, Peterson JD, Montano EA, Dongari-Bagtzoglou A, Diaz PI, Strausbaugh LD. 2014. Redefining the human oral mycobiome with improved practices in amplicon-based taxonomy: Discovery of Malassezia as a prominent commensal. PLoS ONE 9: e90899.

Estrada-Gutierrez G, Gomez-Lopez N, Zaga-Clavellina V, Giono-Cerezo S, Espejel-Nuñez A, Gonzalez-Jimenez MA, Espino y Sosa S, Olson DM, Vadillo-Ortega F. 2010. Interaction between pathogenic bacteria and intrauterine leukocytes triggers alternative molecular signaling cascades leading to labor in women. Infect Immun 78: 4792-4799.

Findley K, Oh J, Yang J, Conlan S, Deming C, Meyer JA, Schoenfeld D, Nomicos E, Park M, Kong HH, et al. 2013. Topographic diversity of fungal and bacterial communities in human skin. Nature 498: 367-370.

Ghannoum MA, Jurevic RJ, Mukherjee PK, Cui F, Sikaroodi M, Naqvi A, Gillevet PM. 2010. Characterization of the oral fungal microbiome (mycobiome) in healthy individuals. PLoS Pathog 6: e1000713.

Gilbert JA, Hughes M. 2011. Gene expression profiling: Metatranscriptomics. Methods Mol Biol 733: 195-205.
Heinsbroek SE, Oei A, Roelofs JJTH, Dhawan S, te Velde A, Gordon S, de Jonge WJ. 2012. Genetic deletion of dectin1 does not affect the course of murine experimental colitis. BMC Gastroenterol 12: 33.

Hilty M, Burke C, Pedro H, Cardenas P, Bush A, Bossley C, Davies J, Ervine A, Poulter L, Pachter L, et al. 2010. Disordered microbial communities in asthmatic airways. PLOS ONE 5: e8578.

Hoffmann C, Dollive S, Grunberg S, Chen J, Li H, Wu GD, Lewis JD, Bushman FD. 2013. Archaea and fungi of the human gut microbiome: Correlations with diet and bacterial residents. PLoS ONE 8: e66019.

Huse SM, Young VB, Morrison HG, Antonopoulos DA, Kwon J, Dalal S, Arrieta R, Hubert NA, Shen L, Vineis $\mathrm{JH}$, et al. 2014. Comparison of brush and biopsy sampling methods of the ileal pouch for assessment of mucosa-associated microbiota of human subjects. Microbiome 2: 5 .

Iatta R, Napoli C, Borghi E, Montagna MT. 2009. Rare mycoses of the oral cavity: A literature epidemiologic review. Oral Surg Oral Med Oral Pathol Oral Radiol Endod 108: 647-655.

Iliev ID, Funari VA, Taylor KD, Nguyen Q, Reyes CN, Strom SP, Brown J, Becker CA, Fleshner PR, Dubinsky M, et al. 2012. Interactions between commensal fungi and the C-type lectin receptor Dectin-1 influence colitis. Science 336: 1314-1317.

Jabra-Rizk MA, Ferreira SM, Sabet M, Falkler WA, Merz WG, Meiller TF. 2001. Recovery of Candida dubliniensis and other yeasts from human immunodeficiency virusassociated periodontal lesions. J Clin Microbiol 39: 45204522.

Johnston BC, Supina AL, Vohra S. 2006. Probiotics for pediatric antibiotic-associated diarrhea: A meta-analysis of randomized placebo-controlled trials. CMAJ 175: $377-$ 383.

Kaufman DA, Gurka MJ, Hazen KC, Boyle R, Robinson M, Grossman LB. 2006. Patterns of fungal colonization in preterm infants weighing less than 1000 grams at birth. Pediatr Infect Dis J 25: 733-737.

Kõljalg U, Larsson K-H, Abarenkov K, Nilsson RH, Alexander IJ, Eberhardt U, Erland S, Høiland K, Kjøller R, Larsson E, et al. 2005. UNITE: A database providing webbased methods for the molecular identification of ectomycorrhizal fungi. New Phytol 166: 1063-1068.

LaTuga MS, Ellis JC, Cotton CM, Goldberg RN, Wynn JL, Jackson RB, Seed PC. 2011. Beyond bacteria: A study of the enteric microbial consortium in extremely low birth weight infants. PLoS ONE 6: e27858.

Lynch SV, Bruce KD. 2013. The cystic fibrosis airway microbiome. Cold Spring Harb Perspect Med 3: a009738.

Ma B, Forney LJ, Ravel J. 2012. Vaginal microbiome: Rethinking health and disease. Annu Rev Microbiol 66: 371-389.

Mahé S, Duhamel M, Le Calvez T, Guillot L, Sarbu L, Bretaudeau A, Collin O, Dufresne A, Kiers ET, Vandenkoornhuyse P. 2012. PHYMYCO-DB: A curated database for analyses of fungal diversity and evolution. PLOS ONE 7: e43117.

Manian FA, Bryant A. 2013. Does Candida species overgrowth protect against Clostridium difficile infection? Clin Infect Dis 56: 464-465. 
P.C. Seed

Mitra S, Rupek P, Richter DC, Urich T, Gilbert JA, Meyer F, Wilke A, Huson DH. 2011. Functional analysis of metagenomes and metatranscriptomes using SEED and KEGG. BMC Bioinformatics 12: S21.

Moyes DL, Naglik JR. 2012. The mycobiome: Influencing IBD severity. Cell Host Microbe 11: 551-552.

Mukherjee PK, Chandra J, Retuerto M, Sikaroodi M, Brown RE, Jurevic R, Salata RA, Lederman MM, Gillevet PM, Ghannoum MA. 2014. Oral mycobiome analysis of HIVinfected patients: Identification of Pichia as an antagonist of opportunistic fungi. PLoS Pathog 10: e1003996.

Palmer C, Bik EM, DiGiulio DB, Relman DA, Brown PO. 2007. Development of the human infant intestinal microbiota. PLoS Biol 5: e177.

Park HK, Ha M-H, Park S-G, Kim MN, Kim BJ, Kim W 2012. Characterization of the fungal microbiota (mycobiome) in healthy and dandruff-afflicted human scalps. PLoS ONE 7: e32847.

Pflughoeft KJ, Versalovic J. 2012. Human microbiome in health and disease. Annu Rev Pathol 7: 99-122.

Pothoulakis C. 2009. Review article: Anti-inflammatory mechanisms of action of Saccharomyces boulardii. Aliment Pharmacol Ther 30: 826-833.

Pragman AA, Kim HB, Reilly CS, Wendt C, Isaacson RE. 2012. The lung microbiome in moderate and severe chronic obstructive pulmonary disease. PLOS ONE 7: e47305.

Pruesse E, Quast C, Knittel K, Fuchs BM, Ludwig W, Peplies J, Glöckner FO. 2007. SILVA: A comprehensive online resource for quality checked and aligned ribosomal RNA sequence data compatible with ARB. Nucleic Acids Res 35: 7188-7196.

Qamar A, Aboudola S, Warny M, Michetti P, Pothoulakis C, LaMont JT, Kelly CP. 2001. Saccharomyces boulardii stimulates intestinal immunoglobulin A immune response to Clostridium difficile toxin A in mice. Infect Immun 69: 2762-2765.

Qin J, Li R, Raes J, Arumugam M, Burgdorf KS, Manichanh C, Nielsen T, Pons N, Levenez F, Yamada T, et al. 2010. A human gut microbial gene catalogue established by metagenomic sequencing. Nature 464: 59-65.
Ravel J, Gajer P, Abdo Z, Schneider GM, Koenig SSK, McCulle SL, Karlebach S, Gorle R, Russell J, Tacket $\mathrm{CO}$, et al. 2011. Vaginal microbiome of reproductiveage women. Proc Natl Acad Sci 108: 4680-4687.

Salonen JH, Richardson MD, Gallacher K, Issakainen J, Helenius H, Lehtonen OP, Nikoskelainen J. 2000. Fungal colonization of haematological patients receiving cytotoxic chemotherapy: Emergence of azole-resistant Saccharomyces cerevisiae. J Hosp Infect 45: 293-301.

Santamaria M, Fosso B, Consiglio A, De Caro G, Grillo G, Licciulli F, Liuni S, Marzano M, Alonso-Alemany D, Valiente G, et al. 2012. Reference databases for taxonomic assignment in metagenomics. Brief Bioinform 13: $682-$ 695.

Schloss PD, Westcott SL, Ryabin T, Hall JR, Hartmann M, Hollister EB, Lesniewski RA, Oakley BB, Parks DH, Robinson CJ, et al. 2009. Introducing mothur: Open-source, platform-independent, community-supported software for describing and comparing microbial communities. Appl Env Microbiol 75: 7537-7541.

Schuster GS. 1999. Oral flora and pathogenic organisms. Infect Dis Clin North Am 13: 757-774.

Tang C, Kamiya T, Kadoki M, Iwakura Y. 2012. A novel role of Dectin-1 signaling in promoting intestinal inflammation. Cytokine 59: 516.

Tung JM, Dolovich LR, Lee CH. 2009. Prevention of Clostridium difficile infection with Saccharomyces boulardii: A systematic review. Can J Gastroenterol 23: 817-821.

Wade WG. 2013. The oral microbiome in health and disease. Pharmacol Res 69: 137-143.

Walsh TJ, Dixon D. 1996. Spectrum of mycoses. In Medical microbiology, 4th ed. (ed. Baron S). University of Texas, Galveston, TX.

White JR, Maddox C, White O, Angiuoli SV, Fricke WF. 2013. CloVR-ITS: Automated internal transcribed spacer amplicon sequence analysis pipeline for the characterization of fungal microbiota. Microbiome 1: 6 .

Williams DW, Lewis MA. 2000. Isolation and identification of Candida from the oral cavity. Oral Dis 6: 3-11.

Zaura E, Keijser BJF, Huse SM, Crielaard W. 2009. Defining the healthy "core microbiome" of oral microbial communities. BMC Microbiol 9: 259. 


\section{$\&_{\mathrm{CSH}}^{\infty} \&$ Cold Spring Harbor

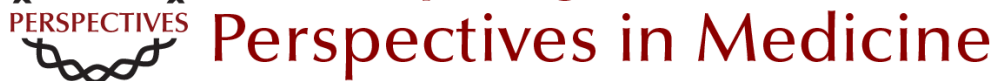

\section{The Human Mycobiome}

Patrick C. Seed

Cold Spring Harb Perspect Med 2015; doi: 10.1101/cshperspect.a019810 originally published online November 10, 2014

\section{Subject Collection Human Fungal Pathogens}

\section{Evolutionary Perspectives on Human Fungal Pathogens John W. Taylor}

Black Molds and Melanized Yeasts Pathogenic to Humans Anuradha Chowdhary, John Perfect and G. Sybren de Hoog

Fungal Pathogens: Survival and Replication within Macrophages Andrew S. Gilbert, Robert T. Wheeler and Robin C. May

Innate Defense against Fungal Pathogens Rebecca A. Drummond, Sarah L. Gaffen, Amy G. Hise, et al.

Antifungal Pharmacokinetics and Pharmacodynamics Alexander J. Lepak and David R. Andes

Human Fungal Pathogens of Mucorales and Entomophthorales

Leonel Mendoza, Raquel Vilela, Kerstin Voelz, et al.

Functional Profiling of Human Fungal Pathogen Genomes

Alexi I. Goranov and Hiten D. Madhani

Aspergillus fumigatus and Related Species Janyce A. Sugui, Kyung J. Kwon-Chung, Praveen R. Juvvadi, et al.
Thermally Dimorphic Human Fungal Pathogens-Polyphyletic Pathogens with a Convergent

Pathogenicity Trait Anita Sil and Alex Andrianopoulos

Mechanisms of Antifungal Drug Resistance Leah E. Cowen, Dominique Sanglard, Susan J. Howard, et al.

Treatment Principles for Candida and Cryptococcus Laura C. Whitney and Tihana Bicanic

The Human Mycobiome Patrick C. Seed

Treatment Principles for the Management of Mold Infections

Dimitrios P. Kontoyiannis and Russell E. Lewis

Adaptive Immunity to Fungi Akash Verma, Marcel Wüthrich, George Deepe, et al.

The Candida Pathogenic Species Complex Siobhán A. Turner and Geraldine Butler

Fungal Morphogenesis Xiaorong Lin, J. Andrew Alspaugh, Haoping Liu, et al.

For additional articles in this collection, see http://perspectivesinmedicine.cshlp.org/cgi/collection/ 